\title{
Memory for general and specific sentences*
}

\author{
SAMUEL J. THIOS \\ Denison University, Granville, Ohio 43023
}

\begin{abstract}
Cued recall indicated that memory was better for sentences containing specific verbs (e.g., scratched) than for sentences containing general verbs (e.g., injured). When synonymic verb responses were included, however, the general-specific difference was eliminated. Also, for complete sentence recall, subject nouns were better retrieval cues than verbs or object nouns.
\end{abstract}

There have been, in recent years, a number of studies which have investigated the role of the verb in memory for sentences. In most of these studies where sentence recall was prompted with the subject, the verb, or the object as a cue, the verb typically was relatively ineffective as a retrieval cue. In one recent study Wearing (1972) suggests several possible reasons why the verb often is a poor retrieval cue. One reason is that, when a sentence is encoded, it may be stored such that the subject and object nouns are maintained as separate events and the verb is broken into components which are linked to these nouns. That is, the verb may indicate those aspects or qualities of the interacting nouns which are important for the intended communication. Thus, the verb may be stored in memory only indirectly.

The present study investigated memory for sentences which contained either general or specific verbs. It was predicted that specific sentences would be remembered better than general sentences because they specify more attributes of the interacting nouns. For example, the general sentence, "The uncle made the toy," indicates that a person (uncle) caused some object (toy) to come into existence. In the specific sentence, "The uncle carved the toy," the same information as above is indicated. However, in addition, the specific sentence implies that the toy was made of a substance capable of being carved (probably wood) and that a sharp instrument was used (probably a knife). It was hypothesized that the specific verb, by indicating additional properties of the interacting nouns, would provide more information which could potentially aid Ss during later sentence retrieval.

A second purpose of this study was to further investigate the relative effectiveness of different types of retrieval cues. Thus, some Ss were cued with the subject noun, some were cued with the verb, and some were cued with the object noun.

\section{METHOD}

\section{Subjects}

The Ss were 60 undergraduates at the University of Virginia.

*This research was supported by National Science Foundation Research Grant GB-22664 to $L$. Starling $R$ eid and by a grant to the author from the Denison University Faculty Development Activities Program. Thanks are due Bill Stehle and Larry Giordano, who assisted in data collection and a nalysis.
They were naive with respect to verbal learning experiments and were either paid for participation or received credit for a course requirement.

\section{Stimulus Materials and Apparatus}

The sentence materials were constructed in the following way. For each of 22 noun pairs, both a general and a specific verb were selected. In each instance, the specific verb was chosen to specify the same type of activity indicated by the general verb. For example, "The hunter improved the cottage" is a general sentence, whereas "The hunter painted the cottage" is a specific sentence. In this instance, painting, of course, represents only one of many possible ways in which the cottage could have been improved. In selecting the sentences, an attempt was made to minimize associative connections between words both within sentences and between sentences. Prior to the experiment, an independent group of Ss had rated the general and specific sentences to be approximately equal in ease of comprehension. Also, the sentence nouns were rated on a 7-point scale for image value. The subject nouns and object nouns were rated equal in image value (mean $=4.12$ for subject nouns and 4.18 for object nouns).

All sentences and test cues were photographed on $35-\mathrm{mm}$ film and mounted on slides. They were projected onto a large screen by a slide projector. Timing sequences were controlled by an electronic timer.

\section{Experimental Design}

Verb relationship (either general or specific) was a within-Ss variable. Twenty Ss were tested under each cue condition and all Ss were presented with 26 sentences. The first four sentences were buffers and were included to control for primacy effects. Recency was controlled by insuring that none of the last four input sentences was tested in any of the first four test positions. For the 22 critical sentences (11 general, 11 specific), one input order was determined, such that the mean list position was approximately equal for general and specific sentences. A second list order was formed by flip-flopping the general and specific sentences in the first order. There were two orders of test cues In both orders the mean list position for general and specific sentence cues was approximately the same. Ss were tested in groups of five. The experimental condition for each group was determined randomly as each group reported to the laboratory.

\section{Procedure}

Both the sentences and test cues were presented visually. During the 10 -sec presentation time, Ss rated each sentence on a 6-point scale as to how easy or how difficult it was to comprehend the sentence. This was done to help insure that Ss paid attention to all sentences. Immediately following presentation, Ss were presented with the cues for recall and each cue was presented for $20 \mathrm{sec}$. All Ss were presented with a booklet for recall. On each page of the booklet was the sentence frame: The When presented with the cue, Ss wrote the cue in the appropriate blank and then filled in the remainder of the 
sentences. After filling in the two missing parts of the sentence they rated each part on a 6-point scale as to how confident they were in their responses. They also were required to respond to every cue, even if they had to guess. At the beginning of the experiment, they were told they later would be presented with one word from each sentence to help them recall; however, they did not know which cue they would receive until time of testing.

\section{RESULTS AND DISCUSSION}

The data for complete sentence recall are summarized in Table 1 , where mean proportion correct sentence recall for general and specific sentences is presented as a function of type of cue. Each proportion is based on 440 observations. The data were analyzed by a two-way analysis of variance, with one fixed factor (type of cue) and one random factor (verb relationship). The results indicated a highly significant main effect of verb relationship $[F(1,57)=64.70, p<.001, \quad S E=.46]$. Thus, clearly, specific sentences were better recalled than general sentences. The results also indicated a marginally significant main effect of cue type $[F(2,53)=$ $2.83, .05<\mathrm{p}<.10, \quad \mathrm{SE}=.16$. Subsequent $t$ tests indicated that the subject was a better cue than either the object $[\mathrm{t}(19)=4.53, \mathrm{p}<.01, \mathrm{SE}=.26]$ or the verb $[\mathrm{t}(19)=7.09, \mathrm{p}<.01, \mathrm{SE}=.28]$ and that the lat ter two did not differ significantly. The interaction did not approach significance.

The finding that the subject noun was the best cue for these sentences is consistent with the results of Horowitz and Prytulak (1969), who used similar sentences and also found that the subject noun was the best cue for sentence recall. In interpreting their results, these authors made use of the "theme-rheme" or "topic-comment" distinction which has been discussed by several investigators (e.g., Halliday, 1967; Hockett, 1966). Thus, an English clause consists of a "logical or psychological" subject (the theme) and the body of the message (or rheme). Both the theme and rheme are high-order constituents of the clause. Thus, the subject is the best cue, since it is the complete theme, whereas the verb or the object represents only part of the rheme. The results of the present study are consistent with such an interpretation. Until further evidence is available, however, the small advantage of the subject noun as a cue $(6 \%)$ should be interpreted with caution. This is

Table 1

Mean Proportion Correct Sentence Recall for General and Specific Sentences as a Function of Type of Cue

\begin{tabular}{lllllr}
\hline \multirow{2}{*}{$\begin{array}{c}\text { Cue } \\
\text { Type }\end{array}$} & \multicolumn{4}{c}{ Sentence } & Type \\
\cline { 2 - 5 } & \multicolumn{2}{c}{ General } & \multicolumn{2}{c}{ Specific } & Mean \\
\hline Subject & .28 & $(.47)$ & .45 & $(.50)$ & .37 \\
Verb & .20 & & .35 & & .28 \\
Object & .24 & $(.36)$ & .38 & $(.40)$ & .31 \\
Mean & .24 & & .40 & & \\
\hline
\end{tabular}

Note-Values in parentheses indicate correct sentence recall when synonymic verb responses are included. especially the case since recent evidence (e.g., Danks \& Sorce, 1973; James, 1972; Perfetti \& Goldman, 1973) indicates that, when investigating memory for sentences, image value of subject and object nouns must be carefully considered as well as syntactical role. For example, Perfetti and Goldman suggest that, when sentences are presented individually without context, there is no difference between subject and object prompt effectiveness if the two nouns are equal and high in rated imagery.

As for the finding that specific sentences are better recalled than general sentences, several points appear relevant to the interpretation of this result. First, the data appear to support the hypothesis that specific verbs specify additional attributes of the interacting nouns and that this added information results in improved recall performance.

Before discussing this finding further, however, it should be noted that inspection of Ss' answer booklets indicated that in a number of cases the subject and object nouns were correct but that Ss were not given credit for sentence recall since the verb was incorrect. However, it also was apparent that in many cases the verb "intrusion" was very close in meaning to the original verb. Thus, the data were rescored in the following manner. A sentence was scored as correct if both nouns were correct and the verb intrusion was judged to be synonymous with the original verb. A group of 20 Denison undergraduates rated the verb intrusions on a 6-point scale as to whether or not the sentence with the new verb could be considered to be synonymous with the original sentence. The data were then rescored counting as correct those verb intrusions which received a rating of 4,5 , or 6 on the 6-point scale and were, thus, rated as synonymous. As an example, for the general sentence "The leader provoked the hostage," "angered" was rated as synonymous, whereas "scared" and "called" were not. The resulting data from this analysis are represented by the values in parentheses in Table 1. It is apparent from Table 1 that verb synonyms are much more common for general sentences and, when included, $\mathrm{t}$ tests indicated no significant differences for either the subject-cue or object-cue condition.

One interpretation of this finding which appears consistent with the present position is as follows. When an $S$ is presented with a sentence, he "abstracts" the essential information contained in the sentence. This "abstracting" process has recently been described by, among others, Zangwill (1972). With general sentences, fewer noun attributes are specified and, therefore, less information is abstracted. At time of recall, S's task essentially is to reconstruct the sentence based on his previously abstracted information. The $S$ is, thus, likely to substitute a similar or synonymous response, since the general relationship specified by the sentence can be realized by many different verbs. Returning to the earlier example, "The uncle made the toy," the uncle 
caused the toy to come into existence is the essence of the abstracted information. This can be realized by verbs such as "built," "constructed," and many others, as well as the verb "made." Thus, recall will be relatively depressed when scored by a verbatim criterion. On the other hand, when $S$ is presented with a specific sentence, the same abstracting process takes place but additional information is present. The additional attributes indicated by the specific verb thus make it more likely that one will be able to remember the exact verb.

Although the above interpretation is consistent with the obtained results, there is an alternative interpretation which should be considered. That is, it may be the case that specific sentences are better recalled because they are easier to image. Paivio (1971) recently has extended the dual-coding model to memory for connected discourse. Thus, for concrete sentences such as "The boy hit the girl," imagery is likely to predominate. On the other hand, for abstract sentences such as "The theory has predictive power," the sentence is more likely to be stored in its verbal form. In the present study, it is important to note that the sentence nouns were constant for both general and specific sentences; however, it certainly is still possible for the sentences to differ along an imagery dimension as a function of verb changes. Therefore, imagery ratings were obtained from a separate group of 20 Denison undergraduates. They rated each sentence on a 4-point scale as to how easy or difficult it was to get an image for that sentence. Overall, these data indicate that specific sentences are rated slightly higher in image value than general sentences. The mean value was 2.3 for specific sentences as compared with 2.1 for general sentences. Although this difference is small, it is in the direction predicted by the imagery hypothesis. A closer examination of the ratings of individual sentences, however, revealed an interesting pattern: Out of the 22 sentences, 7 of the general and specific sentences were rated equal in imagery and, for 3 of the sentences, the general form was rated slightly higher in imagery than the specific form. Thus, to get an approximation of the possible contribution of imagery, the data for only these 10 sentences were rescored. When scored in this fashion, the resulting proportions remain almost unchanged from those proportions presented in Table 1 . The subject noun still is a better retrieval cue than the object noun and, more importantly, the general-specific difference is not diminished. Thus, while imagery may be an important variable in sentence memory, it does not seem to be accounting for the general-specific differences found in the present study.

In short, the present study provides additional support for the contention previously expressed by several investigators that intrusion analysis can be an important source of information in sentence memory experiments. The fact that the general-specific difference is eliminated when synonymic verb intrusions are included indicates that Ss were "remembering" the basic ideas communicated in the general sentences. This finding, of course, is similar to the "memory for gist" phenomenon described by Fillenbaum (1966) and further supports the observation that people often fail to recall a sentence verbatim but do recall an acceptable paraphrase of the sentence. This finding has been well documented by several investigators (e.g., Clark \& Clark, 1968; Fillenbaum, 1966; Mehler, 1963; Miller, 1962). When verbatim recall is measured, however, sentences containing specific verbs specify additional noun features and produce better recall. Although the noun features specified by general verbs make it more difficult to retrieve the exact verb, it seems clear that Ss often remember the essential meaning from general sentences.

\section{REFERENCES}

Clark, H. H., \& Clark, E. V. Semantic distinctions and memory for complex sentences. Quarterly Journal of Experimental Psychology, 1968, 20, 129-138.

Danks, J. H., \& Sorce, P. A. Imagery and deep structure in the prompted recall of passive sentences. Journal of Verbal Learning \& Verbal Behavior, 1973, 12, 114-117.

Fillenbaum, S. Memory for gist: Some relevant variables. Language \& Speech, 1966, 9, 217-227.

Halliday, M. A. K. Notes on transitivity and theme in English: II. Journal of Linguistics, 1967, 3, 199-244.

Hocket $t, C$. F. The problem of universals in language. In J. H. Greenberg (Ed.), Universals of language. 2 nd ed. Cambridge: MIT Press, 1966 .

Horowitz, L. M. \& Prytulak, L. S. Redintegrative memory. Psychological Review, 1969, 76, 519-531.

James, $C$. T. Theme and imagery in the recall of active and passive sentences. Journal of Verbal Learning \& Verbal Behavior, 1972, 11, 205-211.

Mehler, J. Some effects of grammatical transformations on the recall of English sentences. Journal of Verbal Learning \& Verbal Behavior, 1963, 2, 346-351.

Miller, G. A. Some psychological studies of grammar. American Psychologist, 1962, 17, 748-762.

Paivio, A. Imagery and language. In S. J. Segal (Ed.), Imagery: Current cognitive approaches. New York: Academic Press, 1971.

Perfetti, C. A., \& Goldman, S. Structural variables in the retrieval of sentences from paragraphs. Paper presented at the meeting of the Midwestern Psychological Association, Chicago, May 1973 .

Wearing, A. J. Remembering complex sentences. Quarterly Journal of Experimental Psychology, 1972, 24, 77-86.

Zangwill, $O$. L. Remembering revisited. Quarterly Journal of Experimental Psychology, 1972, 24, 123-138.

(R eceived for publication March 11, 1974; revision received June $10,1974$. ) 